\title{
Recurrent asthma exacerbations: What else could it be?
}

\author{
Hawa Edriss MD, Brian Williams MD
}

\begin{abstract}
A 24-year-old woman with a longstanding diagnosis of asthma presented with worsening shortness of breath, aphonia, and dry cough after she was exposed to cooking fumes at work. She was hospitalized and treated for an acute asthma exacerbation. The patient has had frequent emergency center visits for the same complaints for which she was treated with corticosteroids and bronchodilators and discharged home to continue using her inhaler (salmeterol $50 \mathrm{mcg} /$ fluticasone $500 \mathrm{mcg}$ ). She underwent direct laryngoscopic examination (LGS) to evaluate her aphonia and subsequently videoscopic examination which revealed broad-based papilloma-like lesions on the anterior commissure of the glottis and $0.5 \mathrm{~cm}$ immediately below the vocal cord in the subglottic area. These lesions were surgically removed with a microdebrider by an otolaryngologist and sent to histopathology which revealed layers of squamous cells with viral cytopathic effects, including koilocytes and high grade dysplasia (positive p16 staining). Immunohistochemical staining was positive for HPV subtypes 6 .
\end{abstract}

The patient improved, but her symptoms recurred one year later. Direct LGS examination demonstrated recurrent laryngeal and subglottic papillomatosis with separate lesions on the left ventricle, the anterior commissure, and the right vocal fold. A total of $0.5 \mathrm{ml}$ of cidofovir was injected into these lesions. Three microdebridements of recurrent laryngeal lesions and two injections of cidofovir were performed at intervals of about a year each. Two years later, she presented again with shortness of breath with minimal exertion but no stridor or aphonia. Flexible bronchoscopic examination showed a small laryngeal lesion

Corresponding author: Hawa Edriss

Contact Information: Hawa.edriss@ttuhsc.edu DOI: $10.12746 /$ swrccc.v6i22.443 of about $3 \mathrm{~mm}$ in size. However, she had developed two mid-tracheal papillomatous structures of about $3 \times 1 \mathrm{~cm}$ and $2 \times 1 \mathrm{~cm}$ in size (Figure 1); both were treated with cryo-application (Figure 2). Laser excision will be considered if these recur again. Intralesional interferon-alpha injection was not performed due to financial issues.

\section{Discussion}

This patient was treated for persistent asthma for several years before she was diagnosed with recurrent respiratory papillomatosis. This is not an uncommon situation since laryngeal and tracheal papillomatosis is rare, especially in adults. The diagnosis can be delayed and/or incorrect due to symptom similarities to other more common diseases, such as asthma, gastro-esophageal reflux, infections, and benign or malignant laryngeal or tracheal tumors. The clinical presentation includes hoarseness if it affects the

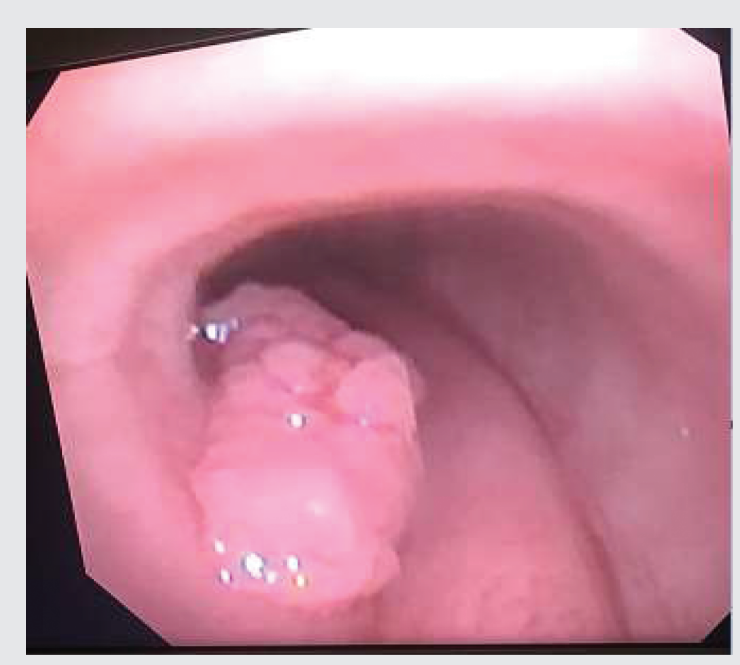

Figure 1. A characteristic HPV warty growth at mid-trachea level of approximately $1 \times 3 \mathrm{~cm}$ in size 


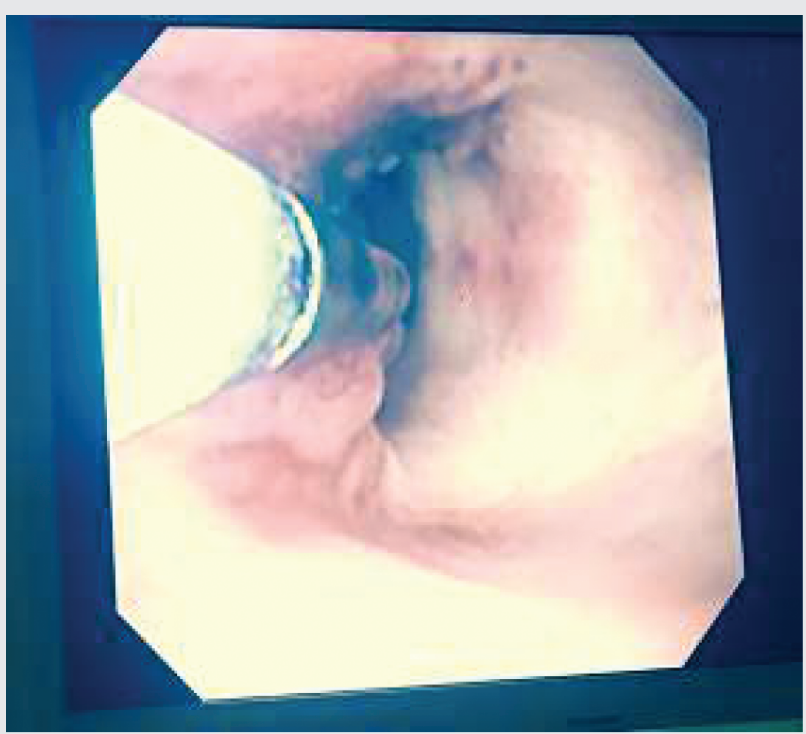

Figure 2. Cryotherapy application for tracheal papillomatosis debulking.

larynx; other symptoms include cough, dyspnea, foreign body sensation in the throat, inspiratory wheeze, and stridor. ${ }^{1}$ Laryngoscopy or bronchoscopy is necessary for any patient with voice changes or hoarseness to confirm the diagnosis as it demonstrates characteristic warty growths. Any region of the respiratory tract can be involved. However, laryngeal involvement occurs in more than $95 \%$ of patients. Rarely, the trachea is involved without laryngeal lesions. It is mandatory to histologically confirm respiratory papillomatosis; biopsy is indicated for viral typing and to rule out malignant transformation. ${ }^{2}$

Recurrent respiratory papillomatosis is a warty growth caused by human papilloma virus (HPV), the majority of cases caused by HPV 6 and $11 .^{2}$ The estimated incidence in patients older than 15 years is approximately 1.8 cases per 100,000 population. ${ }^{3}$
The risk of transmission of HPV in adults with papillomatosis is unclear, but sexual transmission is likely a factor. Treatment often requires repeated debulking of the warts by laser or microdebridement coupled with intralesional cidofovir therapy in patients with moderate or severe disease. ${ }^{4}$ Interferon treatment appears to slow the rate of growth. Eventually, some patients enter remission. In $2-5 \%$ of patients, respiratory papillomas undergo malignant transformation to squamous cell carcinoma, and the prognosis for these patients is very poor.

Keywords: papillomatosis, human papilloma virus, larynx, trachea

From: The Department of Internal Medicine at Texas Tech University Health Sciences Center, Lubbock, TX (HE) and Covenant Medical Center, Lubbock, TX (BW). Submitted: $11 / 11 / 17$

Accepted: $12 / 8 / 17$

Reviewer: Tam Nguyen MD

Conflicts of interest: none

\section{REFERENCES}

1. Andrus JG, Shapshay SM. Contemporary management of laryngeal papilloma in adults and children. Otolaryngol Clin North Am 2006 Feb; 39(1):135-58.

2. Karatayli-Ozgursoy S, Bishop JA, Hillel A, Akst L, Best SR. Risk factors for dysplasia in recurrent respiratory papillomatosis in an adult and pediatric population. Ann Otol Rhinol Laryngol 2016; 125(3): 235-241.

3. Armstrong LR, Preston EJ, Reichert M, Phillips DL, Nisenbaum R, Todd NW. Incidence and prevalence of recurrent respiratory papillomatosis among children in Atlanta and Seattle. Clin Infect Dis 2000 Jul; 31(1):107-9.

4. Chhetri DK, Shapiro NL. A scheduled protocol for the treatment of juvenile recurrent respiratory papillomatosis with intralesional cidofovir. Arch Otolaryngol Head Neck Surg 2003 Oct; 129(10):1081-5. 